\title{
Giving Up Control - a Speculative Air Pollution Mask to Reflect on Autonomy and Technology Design
}

\author{
Britta F. Schulte \\ Zuzanna Lechelt \\ Aneesha Singh \\ University College London \\ Gower Street, London, WC1E 6BT \\ britta.schulte.15@ucl.ac.uk, susan.lechelt.15@ucl.ac.uk, \\ aneesha.singh@ucl.ac.uk
}

\section{Abstract}

In many metropolitan cities air pollution regularly exceeds safe levels, with numerous consequences for health and well-being. Current technological solutions often aim to give users control over their air pollution exposure by measuring, processing and sharing data about pollutant levels. We created a speculative face mask that opens and closes autonomously, taking control away from the user. The goal of the speculative design was to highlight the urgency and effect of air pollution on individuals in a tangible and embodied way. Through this critical design object, we question existing solutionist approaches to air pollution and pose questions about autonomy and individual responsibility. In this paper, we share our development process and the conceptual idea behind the mask to inform and inspire other critical objects to address important societal issues at an individual level.

\section{Author Keywords}

Pollution; critical design; design fiction; sustainability

\section{ACM Classification Keywords}

H.5.m. Information interfaces and presentation (e.g. $\mathrm{HCI}$ ): Miscellaneous

\section{Introduction}

Air pollution regularly exceeds safe levels in many big cities as indicated by recent EU [5] and WHO [13] reports. Most technological solutions to this problem measure air pollution and make the data available (e.g. [7]) with the aim of distilling a sense of control in users, e.g. by enabling them to avoid high levels of pollution or use a pollution mask to reduce their exposure. 
I am late. Running around the flat, I try to get all my stuff together. I can hear the SHARKY yelp in its station. It wants to get out. It needs to get air. Well, I need to get air, it needs to get the $\mathrm{Co} 2$ in the air. I could not breathe without it outside. It could not get outside without me. We need each other. And now we need to get out. It is about time. I finally have all my stuff together I think. I hope. I grasp the brace from the holder and put it on. The usual wiggle until it fits and off we go. I open the door. Immediately I can feel SHARKY grow. I get one breath of the foul-smelling air before it manages to cover my face. In the reflection of the glass on the door I can see that it has grown quite big today. I can see its gills flapping hectically while it tries to get all the nutrients to fill its empty reservoirs. I suck happily on this sweet smelling oxygen that makes its way into my mouth. The level is so high at this early stage that I get a bit giddy. I put my glasses on that make the sky look blue again and step outside with a smile.

Figure 1: Excerpt from one of the design fictions
We used critical design to create an air pollution mask that takes control away from the user by opening and closing automatically depending on the localized air pollution levels around the wearer. In contrast with existing masks, where the wearer is in control of the act of limiting their pollution exposure, this mask is controlled by the level of pollution itself. We are interested in the question of whether and how this changed dynamic of control, as well as the increased tangibility the mask lends to air pollution, changes people's perceptions. Does the speculative design make the intangible threat of air pollution more urgent, tangible and pertinent to the individual?

In this paper we present our design process for the mask, and initial insights of people's perceptions of the mask when exhibited at a local festival. We expect that the concept, our framing of the problem, as well as our reflections can inspire discussion and future work around air pollution.

\section{Background}

Newspapers regularly report on causes of air pollution in cities and possible measures to tackle it. However, this has an arguably limited impact on citizens, as in most Western capitals particularly, the threat seems to be abstract and invisible, with few immediate effects on personal health or links to personal behaviour. London based research groups such as London Air from King's College London address the problem by freely distributing data measured for a number of air pollutants to users online and through mobile apps [7]. The information is provided through an easily accessible traffic light system and is personalised to geographical locations of interest to the user. Applications like London Air are highly informative, but we pose the question whether such approaches instill a sense of immediacy and urgency, and provoke people to engage critically with pervasive air pollution?

We instead draw on critical design, which is an approach that is less solution-oriented, but rather aims to develop speculative artefacts that stimulate debate and question current trends or emerging technologies (e.g. $[1,14])$. Critical design has been previously used, with much promise, to enable people to reflect on autonomy in regards to quantifying data $[6,8]$ or in regards to sustainability $[9,10]$. It offers two features that are particularly suited to the problem at hand: firstly it has been used to overcome "solutionism"-the trend of current technological approaches to fix or solve real-world issues (e.g. $[2,3]$ )-by engaging users in a more playful experience, that can lead to unexpected novel ideas or discussions. Secondly, in contrast to other types of activism, critical design does not aim to be "didactic" [4], but stays deliberately ambiguous, encouraging viewers to come to their own conclusions and responses to the issue (compare e.g. [6]).

\section{Development and implementation}

We used a range of design methods to develop the mask. After all authors debated the key conceptual ideas and the target values of the mask together, BS used traditional sketching methods, as well as more innovative design fiction writing methods to design the mask (see Figure 1). Design fiction has been called a "prototyping technique" [9], which can enable designers to look beyond the object itself, at how it is embedded in society. Design fiction writing helped us to conceptualize the aim and design of the mask at an early stage: we decided that the intention of the artefact was to convey the pressing, current threat of 

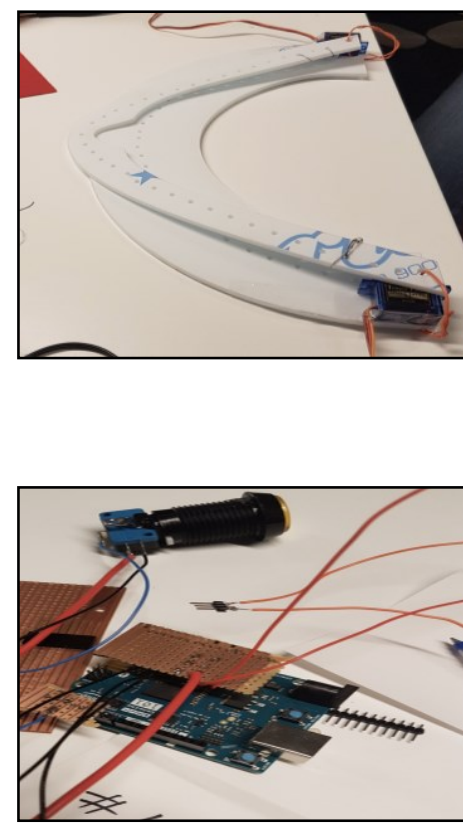

Figure 2: (top) Laser cut mask collar with holes to stitch fabric. (bottom) Final circuit attached to servo to operate mask. air pollution. We further decided that a fabric-based design that might feasibly fit into near-future wear would reflect this intention better than a more futuristic design that might convey the issue of air pollution as a future problem. Through the design fiction writing and sketching process (see Figure 5), the conceptual design for the mask evolved into a collar-inspired artifact. In its resting position (Figure 3, left), the mask took the appearance of a collar. When it was actuated, it unfolded over the wearer's mouth and nose (Figure 3, right). AS and ZL developed a medium-fidelity prototype of the mask, using laser-cut and 3D printed components, fabric, a Genuino 101 and servo motors (Figure 2).

We exhibited our first prototype at a local festival, where we used a Wizard of $\mathrm{Oz}$ approach through which the users could experiment with the mask functionality based on air pollution values, instead of using air pollution sensors to actuate the mask; this explained the function of the mask to the viewer without actually having to move around the city to experience varying pollution levels. Despite the Wizard of Oz approach, it was important that the user did not have direct control of the mask (for example, by pressing a button to change the mask state), but rather could experience the mask being controlled through air pollution values. Therefore, we created an accompanying Bluetooth Low Energy web interface, which controlled the mask state (Figure 3, left) depending on users' input of an air pollution value in line with the Air Quality Index [5]. For example, dangerously high pollution levels actuated a rapid opening of the mask to cover the mannequin's nose, while low values returned it to the collar state.

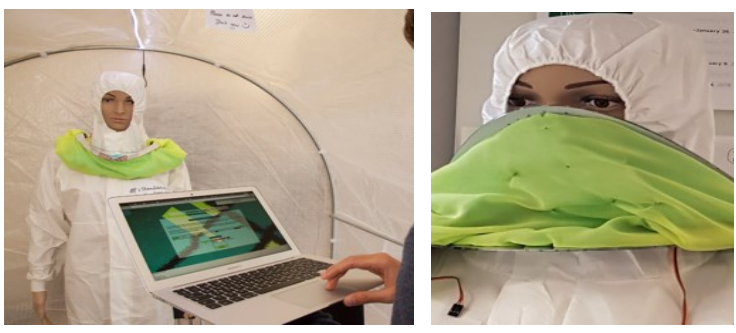

Figure 3: (left) Mannequin wearing mask in closed position laptop showing user interface to control air pollution levels. Picture credit Stuart Keegan, 2017. (right) Mask in open position.

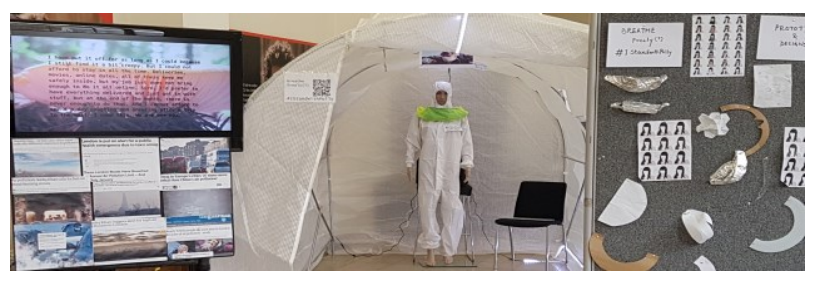

Figure 4: Deployment at festival showing the TV screen to the left with three design fictions of potential mechanic or symbiotic masks. Air pollution news headlines for London were below the TV. In the center of the display is the mannequin in a white plastic tent wearing the mask. To the right are early physical prototypes and sketches from ideation sessions.

\section{First deployment}

The medium-fidelity mask prototype was presented on a fashion mannequin to overcome health and safety issues of sharing a mask and ergonomic issues around its wearability. The display for the festival is shown in Figure 4. Our goal at this exhibition was to explore if the mask could meet its aims by provoking debate around the roles of control and awareness in individual strategies of dealing with air pollution. Visitors were invited to look at the exhibited newspaper headlines and photographs, interact with the mask through the online interface, and chat to researchers. Researchers 


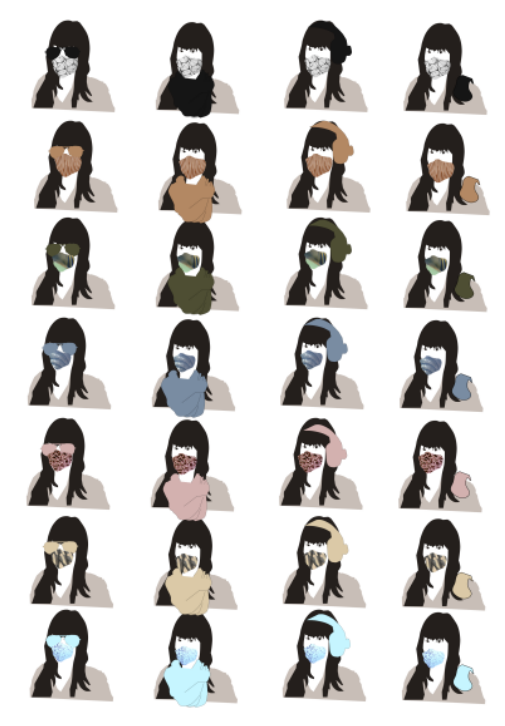

Figure 5: With a strong idea what the mask should do and some impressions of what it might look like we made simple sketches that

became more and more detailed as the project progressed. Putting the mask on a body posed more questions for us: Where could it sit without being too annoying when not in use? How would it open up? How large could/should it be? We experimented with a lot of ideas, some of which are displayed here. observed and made notes on peoples' conversations when interacting with the mask, which formed the basis for the following reflections.

Overall, visitors showed surprise when the mask moved to cover the mannequin's face after they entered high pollution numbers into the web interface. Discussions that followed visitor interactions with the mask often centered on awareness and control over air pollution. Most visitors were London locals who, despite being aware of high levels of air pollution on an abstract and societal level, did not take steps to mediate their exposure to it. They found it difficult to relate to in daily life and practice, because of the invisibility of the problem. For example, one individual reported surprise when his doctor informed him of the immediate impact that running in the polluted city had on his health. Two main reactions emerged, as a number of people commented on control around the self-actuating mechanism of the mask: on one side, people expressed frustrations on the mask taking away control (by acting autonomously based on sensor values); on the other side, the mask was seen as an opportunity to take control over the "unseen" environment by allowing people to change their behaviour. For example, people felt the mask might help them avoid taking routes with high pollution. Moreover, people commented that walking around with a self-actuating mask could raise both their own and other's awareness of the local/geographical aspects of pollution. Overall, the mask invited reflection on pollution as an urgent, tangible problem to be confronted and discussions on what the state of the mask (open vs closed), and hence the environment, meant. Some commented that they felt it could be a form of protest against advancing pollution.
Thus, preliminary observations of visitor discourse at the festival indicate that the mask prototype had the potential to generate varied discussion about people's personal relationships to air pollution and their feeling of control surrounding it. We intend to explore these concepts further in future work.

\section{Future developments and outlook}

Building on insights from the first deployment, we aim to make the experience of the mask more immersive by embedding it in an interactive framework. Moreover, we plan on moving away from the web interface to control the mask, and instead actuating the mask through physical movement on an interactive floor map, which will entail a simplified representation of London Boroughs. Additionally we aim to develop an ergonomic design so that the mask can be worn by participants. Specifically, the mask will open and close depending on the current levels of air pollution in the different city areas, pulled from existing air pollution websites in real time. We hypothesize that the immersive, embodied experience afforded by the act of wearing the mask, and of observing it change while walking the floor map, will further contribute to a more situated, concrete awareness of the air pollution in London. In order to test this, we plan to run a methodical study to qualitatively assess the types of discourse evoked by the interactive experience of wearing the mask.

\section{Acknowledgements}

We thank the Bloomsbury Festival, Roxana Ramirez Herrera, Giulia Barbareschi, Mark Warner, Amid Ayobi for their support and all reviewers for their constructive comments. 


\section{References}

1. Jeffrey Bardzell, Shaowen Bardzell \& Eric Stolterman, 2014. Reading Critical Designs: Supporting Reasoned Interpretations of Critical Design Proceedings, CHI 2014, April 26 - May 01 2014, Toronto, ON, Canada, 1 - 10

2. Mark Blythe, Kristina Andersen, Rachel Clarke \& Peter Wright. 2016. Anti-Solutionist Strategies: Seriously Silly Design Fiction, Proceedings CHI '16, San Jose, CA, USA, 4968 - 4978

3. Mark Blythe, Jamie Steane, Jenny Roe \& Caroline Oliver. 2015. Solutionism, the Game: Design Fictions for Positive Ageing, Proceedings CHI 2015, April 18 - 23, 2015, Seoul, Republic of Korea, 3849 $-3858$

4. James Duggan, Joseph Lindley \& Sarah McNicol. 2017. Near Future School: World Building Beyond a Neoliberal Present with Participatory Design Fictions, Futures, 1 - 9

5. European Commission, Air Quality Standards, online, Retrieved February 27, 2018 from http://ec.europa.eu/environment/air/quality/standa rds.htm

6. William Gaver, Jacob Beaver \& Steve Benford 2003. Ambiguity as a Resource for Design, Proceedings CHI 2013, Ft. Lauderdale, Florida, USA, April 5 - 10, 2003, 1 - 8

7. King's College, London Air Network, online, Retrieved February, 27, 2018 from https://www.londonair.org.uk/LondonAir/MobileApp s/

8. Shaun Lawson, Ben Kirman, Conor Linehan, Tom Feltwell \& Lisa Hopkins. 2015. Problematising Upstream Technology through Speculative Design: the Case of Quantified Cats and Dogs, $\mathrm{CHI}^{\prime} 15$ Proceedings of the 33rd Annual ACM Conference on Human Factors in Computing Systems. ACM, 2663 $-2672$
9. Joseph Lindley \& Paul Coulton. 2016. Pushing the Limits of Design Fiction: The Case for Fictional Research Papers, Proceedings CHI '16, May 07 12, San Jose, CA, USA, $1-12$

10. Stephen Purpura, Victoria Schwanda, Kaiton Williams, William Stubler \& Phoebe Sengers 2011. Fit4Life: The Design of a Persuasive Technology Promoting Healthy Behaviour and Ideal Weight, Proceedings CHI 2011, May 7-12, 2011 Vancouver, BC, Canada.

11. Sebastian Prost, Elke Mattheis \& Manfred Tscheligi. 2015. From Awareness to Empowerment: Using Design Fiction to Explore Paths towards a Sustainable Energy Future, CSCW 2015, March 14 - 18, 2015, Vancouver, BC, Canada, 1649 - 1658

12. Ron Wakkari, Audrey Desjardins, Sabrina Hauser \& Leah Masteri. 2013. A Sustainable Design Fiction: Green Practices, ACM Transactions on ComputerHuman Interactions, 20(4), 1 - 34

13. WHO, Air Quality, online, retrieved February, 27 2018, from http://www.euro.who.int/en/healthtopics/environment-and-health/air-quality/dataand-statistics

14. Johanna Ylipulli, Jenny Kangasvuo, Toni Alatalo \& Timo Ojala. 2016. Chasing Digital Shadows: Exploring Future Hybrid Cities through Anthropological Design Fiction, NordiCHI'16, 23 27, 2016, Gothenburg, Sweden, 1 - 10 